\title{
Non-dissociative adsorption of diatomic molecules on nanoclusters at room temperature
}

\author{
Young Dok Kim *, Gerd Ganteför \\ Department of Physics, University of Konstanz, Universitaetsstrasse 10, D-78464 Konstanz, Germany
}

\begin{abstract}
Experiments using vibrationally resolved ultraviolet photoelectron spectroscopy (UPS) provide evidence that dissociative chemisorption of $\mathrm{N}_{2}$ becomes less stable than the molecular chemisorption on small clusters consisting of less than 10 metal atoms, which can efficiently dissociate diatomic molecules in the bulk form. This result is different from the generally accepted view that undercoordinated atoms are chemically more reactive. Our observation can be rationalized with a less efficient screening of the positive holes in smaller clusters created by a metal to adsorbate charge transfer, caused by the lower number of the directly neighbouring atoms.
\end{abstract}

Nanomaterials are of potential importance in heterogeneous catalysis, since nanoclusters can show unusual catalytic properties, which are not present in the bulk counterparts [1-6]. One of the common characteristics of nanomaterials is that the atoms in nanomaterials have much lower coordination numbers than those of the bulk materials. Ample evidence has been provided in surface chemistry that surface atoms with lower coordination numbers are responsible for enhanced chemical activities. On oxide surfaces, coordinatively unsaturated metal atoms allow catalytic reactions, whereas coordinatively saturated sites are inert [7]. Oxygen vacancies on oxide surfaces

\footnotetext{
${ }^{*}$ Corresponding author. Fax: +49-7531-883888.

E-mail address: young.kim@uni-konstanz.de (Y.D. Kim).
}

are responsible for the catalytic reactions, which do not occur on the stoichiometric oxide surfaces $[8,9]$. On metal single crystal surfaces, atoms at steps, kinks and edges often act as active centres in chemical reactions, whereas terrace atoms do not catalyze the same reactions, which also demonstrates that atoms with lower coordination numbers are chemically more reactive [10,11]. Recent experimental studies on $\mathrm{MoS}_{2}$ and Pd nanoparticles have shown that chemical reactivities on nanoparticles are enhanced on the edges of the particle, which is also evidence for higher catalytic activities of the undercoordinated metal atoms $[12,13]$.

Metal atoms in a cluster consisting of less than 10-15 atoms should have lower coordination numbers of the metal atoms and more opened structures compared to the closely packed metal 
single crystal surfaces (Fig. 1) [14]. Within the concept of the coordination chemistry, one may expect that most of the metal atoms in these small clusters should be chemically active. In the present work, however, we provide evidence that smaller metal clusters can less efficiently dissociate diatomic molecules than the bulk counterparts. This conclusion is based on our experimental observations for three different systems $\left(\mathrm{N}_{2}\right.$ on $\mathrm{Nb}, \mathrm{N}_{2}$ on $\mathrm{W}$, and $\mathrm{H}_{2}$ on $\mathrm{Ti}$ ), suggesting that this may be general phenomenon for many other systems [15]. This result is explained by less efficient screening of the residual positive hole in the metal cluster created by the charge transfer to the adsorbates, analogous to the generally observed final state relaxation effects in photoelectron spectroscopy experiments on nanoparticles.

$\mathrm{M}_{n}^{-}$anions $(\mathrm{M}=\mathrm{W}, \mathrm{Nb})$ are produced with a pulsed arc cluster ion source and mass-selected using a time-of-flight spectrometer (mass resolution $m / \Delta m=400$ ). The temperature of the clusters is estimated to be about room temperature [16]. The mass of the metal anion clusters reacted with $\mathrm{N}_{2}$ molecules is selected by means of a timeof-flight (ToF) mass spectrometer. A selected bunch is irradiated by a UV laser pulse $(h v=4.66 \mathrm{eV})$ and the kinetic energies of the detached electrons are measured using a 'magnetic bottle'-type TOF electron spectrometer with an energy resolution of about $2 \%$.

To shed light on the chemisorption properties of $\mathrm{Nb}$ clusters, the $\mathrm{N}_{2}$ adsorption on $\mathrm{Nb}$ clusters was studied using ultraviolet photoelectron spec-

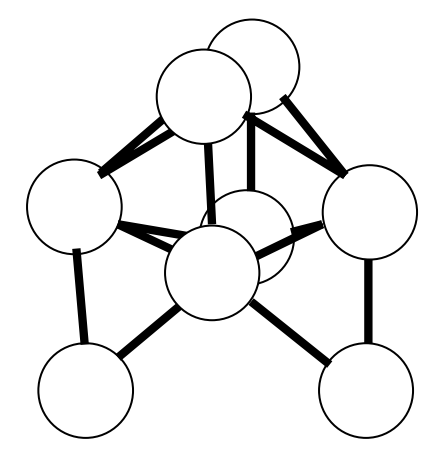

Fig. 1. Structure of an anionic cluster consisting of $\mathrm{Nb}$ atoms. From [14]. troscopy (UPS) (Fig. 2). An advantage of studying $\mathrm{Nb}$ clusters is that previous studies have already thoroughly characterized electronic and geometric structures of $\mathrm{Nb}$ clusters [14-17]. Therefore, correlations between chemical activities and geometric or electronic structures can be determined. Previously, changes of the $\mathrm{N}_{2}$ adsorption reactivities on $\mathrm{Nb}$ clusters were studied and the size dependent reactivity changes were explained in terms of geometric or electronic effects [17-20]. However, spectroscopic measurements on the $\mathrm{N}_{2}$ chemisorbed on $\mathrm{Nb}$ clusters have rarely been carried out, thus the chemisorption mechanism is still questionable [21].

For the $\mathrm{Nb}$ anion clusters with $n=1-6$, relatively narrow peaks at the lowest binding energies

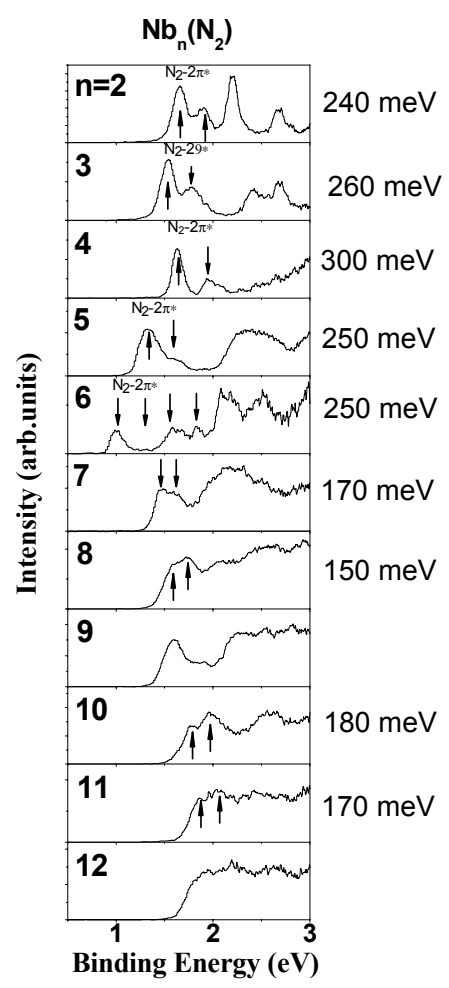

Fig. 2. UPS spectra of the $\mathrm{Nb}_{n} \mathrm{~N}_{2}^{-}$clusters with $n=2-12$. The fine structures of the anion UPS spectra corresponds to the vibrational frequencies of the neutral state. $\mathrm{Nb}$ anion clusters consisting of 8 and 10 atoms are generally less active towards $\mathrm{N}_{2}$ adsorption than other $\mathrm{Nb}$ clusters. By increasing $\mathrm{N}_{2}$ partial pressure in the cluster source, $\mathrm{Nb}_{n} \mathrm{~N}_{2}^{-}$with $n=8,10$ could be synthesized. 
tend to shift to even lower binding energies with increasing number of $\mathrm{Nb}$ atoms (Fig. 2). It is important to note that the energy spacings between neighbouring peaks at the lowest binding energy regime amount to about $250-300 \mathrm{meV}$, which correspond to the vibrational stretching frequency of $\mathrm{N}-\mathrm{N}$ with an end-on structure, i.e. $\mathrm{N}_{2}$ is molecularly chemisorbed with one nitrogen atom attached to the metal cluster $[22,23]$. Note that anion UPS allows the detection of the vibrational frequencies of the neutral species which have an identical geometry to the ground state of the anion, as a consequence of the Franck-Condon principle. From the observation of the vibrational frequency corresponding to the $\mathrm{N}-\mathrm{N}$ stretching at the lower binding energy regime, we conclude that the detachment of an electron from this orbital significantly changes the $\mathrm{N}-\mathrm{N}$ distance. Consequently, the distinct peaks at the binding energy regime of $1.5-2.0 \mathrm{eV}$ for $\mathrm{Nb}_{2} \mathrm{~N}_{2}^{-}$with $n=1-6$ should derive from the $\mathrm{N}_{2}-2 \pi^{*}$ orbitals. Shifts of the $\mathrm{N}_{2}-2 \pi^{*}$ orbitals to lower binding energies with increasing number of $\mathrm{Nb}$ atoms are most likely caused by increased $\mathrm{N}_{2}-\mathrm{Nb}$ interactions. An enhanced $\mathrm{Nb}-\mathrm{N}_{2}$ interaction should lead to a larger splitting of the molecular orbital (MO) formed by the combination of the valence electronic levels of $\mathrm{Nb}$ and $\mathrm{N}_{2}-2 \pi^{*}$ orbitals. A consequence of a larger MO splitting is that the antibonding MO moves towards lower binding energies. It is interesting to note that the $\mathrm{Nb}-\mathrm{N}$ interaction gradually increases with increasing number of $\mathrm{Nb}$ atoms in a cluster.

For $n>6$, peaks at lower binding energies disappear and broader features develop. In contrast to the results from $n<6$, the electron affinity increases with increasing cluster size (Fig. 2). The changes of the UPS pattern from $n=6$ is likely to be caused by a different chemisorption pattern of nitrogen. Energy spacings of about $150-180 \mathrm{meV}$ between different peaks are often observed for $n>6$, which correspond to the $\mathrm{N}-\mathrm{N}$ stretching frequency of the lying-down $\mathrm{N}_{2}$, i.e. two nitrogen atoms are attached to metals [22,23]. It is not surprising that the peaks from $\mathrm{N}_{2}-2 \pi^{*}$ orbitals disappear for $n>6$, considering that the formation of the lying-down structure of the $\mathrm{N}_{2}$ molecules can result in the breakage of the $\pi$-bonding in the $\mathrm{N}-\mathrm{N}$ bonds, and only $\sigma \mathrm{N}-\mathrm{N}$ bonds remain.
Formation of the lying-down structure with much lower $\mathrm{N}-\mathrm{N}$ frequencies for the larger $\mathrm{Nb}$ clusters indicates stronger $\mathrm{N}_{2}-\mathrm{Nb}$ interactions for most of $\mathrm{Nb}_{n}, n>6$ compared to the case with $n<6$. Summarizing the results from $\mathrm{Nb}_{n} \mathrm{~N}_{2}^{-}$with $n=1-10$, the $\mathrm{N}-\mathrm{N}$ bonding tends to be gradually activated with increasing cluster size. Dissociation of $\mathrm{N}_{2}$ does not take place on these $\mathrm{Nb}$ clusters at room temperature.

In addition to $\mathrm{Nb}$ clusters, the molecular adsorption of $\mathrm{N}_{2}$ has also been found for W clusters [24]. As it is displayed in Fig. 3, we observed the vibrational fine structures corresponding to the stretching frequencies of $\mathrm{N}-\mathrm{N}$ on $\mathrm{W}_{n}^{-}$with $n=6,7$ after the reaction of the $\mathrm{W}$ anion clusters with a nitrogen environment. Considering that an indication for the molecule to atom transition was also found for $\mathrm{H}_{2}$ on $\mathrm{Ti}_{n}^{-}$with decreasing cluster size [15], the non-dissociative adsorption of diatomic molecules at room temperature seems to be relevant for many other diatomic molecules on small transition metal clusters.

Because of the pronounced vibrational progression observed, the additional electron most

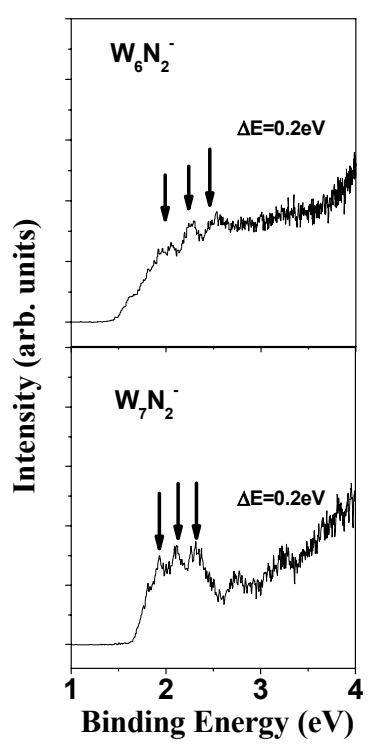

Fig. 3. UPS spectra of various $\mathrm{W}_{n}^{-}$and $\mathrm{W}_{n} \mathrm{~N}_{2}^{-}$clusters $(n=6,7)$ obtained with a photon energy of $4.66 \mathrm{eV} . \mathrm{W}_{n} \mathrm{~N}_{2}^{-}$ clusters were created by exposing tungsten clusters molecular $\left(+\mathrm{N}_{2}\right)$ nitrogen. 
likely occupies an orbital with a strong contribution of the antibinding $2 \pi^{*}$ orbital of $\mathrm{N}_{2}$ on the $\mathrm{Nb}$ and $\mathrm{W}$ anion clusters. It is reasonable to conclude that the propensity of an anion towards dissociative chemisorption should be stronger than that of the neutral counterpart, implying that molecularly bound $\mathrm{N}_{2}$ is the most stable configuration not only for the anionic clusters, but also for the respective neutral species.

Dissociative chemisorption of $\mathrm{N}_{2}$ on metal surfaces is rarely observed under high vacuum conditions at room temperature, since a high activation barrier exists for the dissociative adsorption. In this case, atomically bound nitrogen species can be synthesized by exposing the metal crystals to an atomic nitrogen environment instead of molecular nitrogen. Analogous to this approach in surface chemistry, mass-selected $\mathrm{Nb}$ and $\mathrm{W}$ clusters were exposed to an atomic nitrogen atmosphere in order to gain insights into the chemisorption energetics of these small nanoclusters. As it is illustrated Fig. 4, high intensities for $\mathrm{MN}_{n}^{-}(\mathrm{M}=\mathrm{Nb}$ or $\mathrm{W})$ with $n=$ odd numbers can be observed using atomic nitrogen reagent, indicating that mass-selected metal clusters actually reacted with atomic nitrogen. Using a molecular nitrogen atmosphere, only $\mathrm{MN}_{n}^{-}$with $n=$ even numbers can be observed [24].

In our UPS experiments, the $\mathrm{Nb}_{n} \mathrm{~N}_{2}^{-}$and $\mathrm{W}_{n} \mathrm{~N}_{2}^{-}$ clusters prepared with atomic nitrogen and molecular nitrogen exhibit identical structures, indicating that nitrogen is always molecularly bound on the $\mathrm{Nb}$ and $\mathrm{W}$ clusters, independent of the preparation method. Two nitrogen atoms reacted with the metal clusters spontaneously combine to form di-nitrogen species, implying that molecular nitrogen is thermodynamically more stable than the dissociatively bound nitrogen. An alternative interpretation of this result is that the massselected clusters selectively reacted with small amounts of molecular nitrogen impurities from the atomic nitrogen environments. Following the interpretation that these metal clusters prefer interactions with molecular nitrogen to atomic nitrogen, we also reach the same conclusion that formation of molecularly bound nitrogen is energetically more favourable than dissociative adsorption.
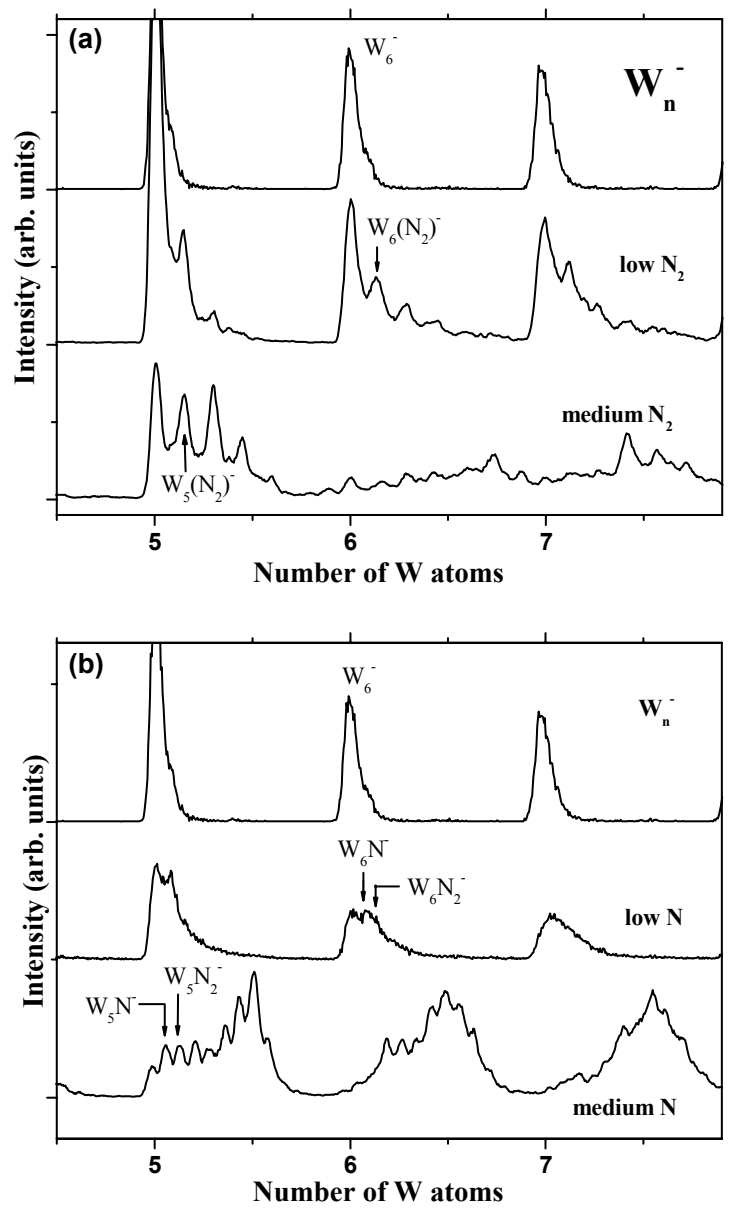

Fig. 4. (a) Mass spectra of tungsten anion clusters before (top trace) and after reaction with molecular nitrogen at two different background pressures of $\mathrm{N}_{2}$. (b) Mass spectra of tungsten anion clusters before (top trace) and after reaction with atomic nitrogen at different background pressures of $\mathrm{N}$.

Early transition metals in bulk form such as Ti, $\mathrm{W}$ and $\mathrm{Nb}$ have strong propensities towards the dissociative chemisorption or the hydride and nitride formation under ambient conditions [23]. Due to lower coordination numbers of the metal atoms in these small clusters, one may expect that these clusters should exhibit higher activities with respect to dissociative chemisorption of the diatomic molecules. However, our experiments demonstrate that these materials become rather inactive for dissociative chemisorption of nitrogen for limited cluster sizes. 
Cluster size dependent changes of reactivities have often been interpreted in terms of geometric structures, i.e. those clusters having specific adsorption sites show increased chemical activities. In this context, it is important to note that the $\mathrm{N}_{2}$ $\mathrm{Nb}$ interaction gradually increases with increasing cluster size in the size range between $n=2-10$, and the chemisorption pattern changes from end-on to the lying-down geometry at $n=7$. Considering the previous results that the $\mathrm{Nb}_{n}^{-}$exhibit compact and low symmetric 3D structures in the size range between $n=3-8$ [13], there is no indication for the activation of $\mathrm{N}_{2}$ being correlated with specific adsorption sites of the $\mathrm{Nb}$ clusters.

Our result can be rationalized by considering changes of the charge transfer from the metal to the antibonding molecular orbital of the adsorbate for different coordination numbers of the metal atoms. For larger particles and bulk crystals, positive holes caused by the charge transfer from the metal to the adsorbate are efficiently screened by neighbouring metal atoms. In contrast, smaller clusters with reduced average numbers of directly neighbouring atoms do not allow such an efficient screening of the residual positive charge. Since a larger charge transfer from the metal atoms to the antibonding orbital of the adsorbate are required for the dissociative chemisorption, dissociation of the diatomic molecules becomes less effective with decreasing cluster size. The reduced screening of the positive residual holes in smaller clusters is evident in photoelectron spectroscopy experiments probing shifts of core-levels as well as valence-levels [2530]. Binding energies of the electrons generally shift to higher energies with decreasing clusters size, since the hole created by the photoelectron emission is not efficiently screened on smaller clusters with lower coordination numbers. We suggest that the final state effects of the hole states in the photoemission experiments are also reflected in the chemistry of nanoclusters. It is important to mention that a close relationship between the work function and the CO dissociation probability was found for transition metals, in line with our suggestion that the binding energy of electrons is crucial for the dissociation of these diatomic molecules [31].
In conclusion, for $\mathrm{N}_{2} / \mathrm{Nb}$ and $\mathrm{N}_{2} / \mathrm{W}$ systems, we observed that dissociative chemisorption becomes less stable compared to the molecular chemisorption on small clusters consisting of early transition metals, which can efficiently dissociate diatomic molecules as bulk crystals. This result is consistent with our previous results on $\mathrm{H}_{2} / \mathrm{Ti}$ system [15]. This behaviour can be rationalized with a less efficient screening of the positive holes in smaller clusters created by the metal to adsorbate charge transfer, due to the lower numbers of the directly neighbouring atoms. Our experimental results contradict the generally accepted belief that undercoordinated sites enhance reactivity. Our experiments suggest that, for this to hold, the amount of metal atoms present should be large enough to also screen the holes that often arise at the surface during reactions. The molecular adsorption of diatomic reagents can also be relevant not only for many other gas phase nanoclusters but also for quantum dots. A better understanding on variations of the chemisorption patterns as a function of cluster size can contribute to determining the optimal cluster sizes for the best performance in various catalytic reactions.

\section{Acknowledgements}

The DFG (Deutsche Forschungsgemeinschaft) is acknowledged for the financial support. Nils Bertram is acknowledged for reading the manuscript.

\section{References}

[1] M. Valden, X. Lai, D.W. Goodman, Science 281 (1998) 1647.

[2] A. Cho, Science 299 (2003) 1684.

[3] A.T. Bell, Science 200 (2003) 1688.

[4] M.R. Zarkin, O. Brickman, D.M. Cox, A.J. Kaldor, Chem. Phys. 88 (1988) 3555.

[5] M.B. Knickelbein, Annu. Rev. Phys. Chem. 50 (1999) 79.

[6] A. Kaldor, D.M. Cox, M.R. Zakin, Adv. Chem. Phys. 70 (1988) 211.

[7] H. Over, Y.D. Kim, A.P. Seitsonen, S. Wendt, E. Lundgren, M. Schmid, P. Varga, A. Morgante, G. Ertl, Science 287 (2000) 1474. 
[8] M.-C. Wu, C.M. Truong, K. Coulter, D.W. Goodman, J. Am. Chem. Soc. 114 (1992) 7565.

[9] M.-C. Wu, C.M. Truong, D.W. Goodman, Phys. Rev. B 46 (1992) 12688.

[10] J.K. Norskov, T. Bligaard, A. Logadottir, S. Bahn, L.B. Hansen, M. Bollinger, H. Bengaard, B. Hammer, Z. Sljivancanin, Y. Xu, S. Dahl, C.J.H. Jacobsen, J. Catal. 209 (2000) 275.

[11] S. Helveg, J.V. Lauritsen, E. Lægsgaard, I. Stensgaard, J.K. Nørskov, B.S. Clausen, H. Topsøe, F. Besenbacher, Phys. Rev. Lett. 84 (2000) 951.

[12] S. Dahl, A. Logadottir, R.C. Egeberg, J.H. Larsen, I. Chorkendorff, E. Törnquist, J.K. Nørskov, Phys. Rev. Lett. 83 (1999) 1814.

[13] S. Schauermann, J. Hoffmann, V. Johanék, J. Hartmann, J. Libuda, H.J. Freund, Angew. Chem. Int. Ed. 41 (2002) 2532.

[14] H. Kietzmann, J. Morenzin, P.S. Bechthold, G. Ganteför, W. Eberhardt, D.-S. Yang, P.A. Hackett, R. Fournier, T. Pang, C. Chen, Phys. Rev. Lett. 77 (1996) 4528.

[15] S. Buckart, N. Blessing, G. Ganteför, Phys. Rev. B 60 (1999) 15639.

[16] C.-Y. Cha, G. Ganteför, W. Eberhardt, Rev. Sci. Instrum. 63 (1992) 5661.

[17] H. Kietzmann, J. Morenzin, P.S. Bechthold, G. Ganteför, W. Eberhart, J. Chem. Phys. 109 (1998) 2275.
[18] J.L. Elkind, F.D. Weiss, J.M. Alford, R.T. Laaksonen, R.E. Smalley, J. Chem. Phys. 88 (1988) 5215.

[19] M.D. Morse, M.E. Geusic, J.R. Heath, R.E. Smalley, J. Chem. Phys. 83 (1985) 2293.

[20] R.L. Whetten, M.R. Zakin, D.M. Cox, D.J. Trevor, A. Kaldor, J. Chem. Phys. 85 (1986) 1697.

[21] D.-S. Yang, M.Z. Zgierski, A. Berces, P.A. Hackett, A. Martinez, D.R. Salahub, Chem. Phys. Lett. 227 (1997) 71.

[22] D.A. King, D.P. Woodruff (Eds.), The Chemical Physics of Solid Surfaces and Heterogeneous Catalysis, vol. 3A, Elsevier Science Publishers B.V., Amsterdam, The Netherlands, 1990.

[23] A. Sellidj, J.L. Erskine, Surf. Sci. 220 (1989) 253.

[24] Y.D. Kim, D. Stolcic, M. Fischer, G. Ganteför, Chem. Phys. Lett. 380 (2003) 359.

[25] T.P. St. Clair, D.W. Goodman, Top. Catal. 13 (2000) 5.

[26] K. Luo, T.P. St. Clair, X. Lai, D.W. Goodman, J. Phys. Chem. B 104 (2000) 3050.

[27] J.A. Rodriguez, M. Kuhn, J. Hrbek, J. Phys. Chem. 100 (1996) 18240.

[28] L-. Zhang, R. Prsaud, T.E. Madey, Phys. Rev. B 56 (1997) 10549.

[29] H.-P. Steinrück, F. Pesty, L. Zhang, T.E. Madey, Phys. Rev. B 51 (1995) 2427.

[30] R.L. Whetten, D.M. Cox, D.J. Trevor, A. Kaldor, Phys. Rev. Lett. 54 (1985) 1494.

[31] R. Hoffman, Rev. Mod. Phys. 60 (1988) 601. 